\title{
Trigonometric spline for medical image interpolation
}

\author{
Samreen Abbas ${ }^{1 *}$, Malik Zawwar Hussain ${ }^{1}$ and Misbah Irshad ${ }^{2}$ \\ ${ }^{I}$ Department of Mathematics, Faculty of Science, University of the Punjab, Lahore, Pakistan. \\ ${ }^{2}$ Department of Mathematics, Faculty of Engineering and Technology, Lahore College for Women University, Lahore, Pakistan.
}

\begin{abstract}
Medical imaging deals with visualising internal structures of the human body to diagnose and treat diseases. Several image processing schemes and algorithms are introduced to overcome the challenges regarding medical image analysis. In this paper, a cubic trigonometric spline scheme with multiple quality control parameters is proposed for medical image processing. In comparison, the proposed scheme is found to be better than both the conventional bilinear and bicubic image processing techniques in terms of peak signal-to-noise ratio, root mean square error and structural similarity index measure.
\end{abstract}

Keywords: Basis functions, image interpolation, medical images, PSNR, SSIM, trigonometric spline.

\section{INTRODUCTION}

In the present era of health care, medical image processing plays a vital role in the entire process from the diagnosis of diseases and treatment planning, to surgical procedures with follow up case studies. Some of the problems in the production of medical images are poor quality, blurriness, unwanted noise, and low contrast. Thus, image enhancement or resolution is necessitated when a surgeon needs to interpret a medical image, which gets deteriorated in quality during the process of acquiring, as it helps to improve image quality or sharpen its features such as edges, boundaries, or contrast to make a graphic display more useful for investigation.

A number of mathematical schemes or algorithms based on spatial domain methods and frequency domain methods are used for digital image processing to improve the visual and objective quality of a medical image (Lau \& Ozawa, 2005; Atoui et al., 2006; Leng et al., 2013).
Most of these algorithms are based on interpolation and manipulation techniques such as histogram manipulation, empirical mode decomposition, and spline and wavelet interpolation techniques.

Splines are considered to be the most suitable kernels in order to attain better quality in medical images, which are widely used in computer aided designing and computer graphics for different tasks. The most popular splines used for medical imaging are convolution based splines. A number of reasons are presented in literature to prove that splines are the perfect fit for medical image processing (Unser, 2002). A comparison of different splines with convolution based splines for medical imaging is also provided, which concludes that splines with a higher degree produce better results as compared to other methods including sinc, linear and convolution based splines (Meijiering, 2000).

In this paper, a cubic trigonometric spline function is proposed for medical image interpolation. Three standard medical images are selected here as test images to evaluate the performance of the proposed scheme in comparison with other conventional image processing techniques, including bilinear and bicubic schemes. Experimental results are conducted both in subjective (visual) and objective measures.

A cubic trigonometric spline of first order geometric continuity $\left(G C^{1}\right)$ with quality control parameters is established. The basis functions and the cubic trigonometric function with their properties are presented. The detailed implementation strategy of the proposed scheme is described and the experiments and simulation results are presented. 


\section{METHODOLOGY}

Trigonometric splines play an important role in electronics and medicine (Qi et al., 2009), shape designing, and data visualisation (Ibraheem et al., 2012; Hussain et al., 2014). A piecewise cubic trigonometric spline of $G C^{1}$ continuity with control parameters has been proposed for medical imaging. The basis functions and the properties are also discussed.

\section{Basis function}

Cubic trigonometric basis functions for given knots $x_{1}<x_{2}<\cdots<x_{n}$ are defined in each of the intervals $I_{i}=\left[x_{i}, x_{i+1}\right] ; i=1, \ldots, n-1$ as follows:

$$
\left.\begin{array}{l}
B_{0, i}(\theta)=(1-\sin \theta)^{2}(1+\sin \theta) \\
B_{1, i}(\theta)=\sin \theta\left(1-\sin ^{2} \theta\right) \\
B_{2, i}(\theta)=\cos \theta\left(1-\cos ^{2} \theta\right) \\
B_{3, i}(\theta)=(1-\cos \theta)^{2}(1+\cos \theta)
\end{array}\right\}
$$

where $\theta=\frac{\pi}{2} \delta, \delta=\left(\frac{x-x_{i}}{h_{i}}\right), \quad h_{i}=x_{i+1}-x_{i} \quad$ and $x \in\left[x_{i}, x_{i+1}\right] ; i=1,2, \ldots, n-1$. It can be observed from equation (1) and Figure 1 that these functions are Bernstein Bézier (BB) like basis functions, so they are called as Bernstein Bézier cubic trigonometric basis functions.

Theorem 1. The Bernstein Bezier cubic trigonometric basis functions defined in equation (1) have the following properties:

a. Non-negativity: $\quad B_{j, i}(\theta) \geq 0 ; i=1,2, \ldots, n-1$,

$$
j=0,1,2,3 \text {. }
$$

b. $\sum_{j=0}^{3} B_{j, i}(\theta)=1 ; i=0,1,2, \ldots, n-1$.

c. Symmetry: $\quad B_{j, i}(\theta)=B_{3-j, i}\left(\frac{\pi}{2}-\theta\right) ; i=1,2, \ldots$,

$$
n-1, j=0,1,2,3 \text {. }
$$

\section{Proof}

(a) For $\theta=\frac{\pi}{2} \delta, \quad \delta=\left(\frac{x-x_{i}}{h_{i}}\right)$, where $h_{i}=x_{i+1}-x_{i}$ and $x \in\left[x_{i}, x_{i+1}\right] ; i=1,2, \ldots, n-1$, then $(1-\sin \theta)^{2}$ $\geq 0,(1+\sin \theta) \geq 0, \sin \theta \geq 0,\left(1-\sin ^{2} \theta\right) \geq 0$, $(1-\cos \theta)^{2} \geq 0, \cos \theta \geq 0,(1+\cos \theta) \geq 0$, and $\left(1-\cos ^{2} \theta\right)$ $\geq 0$. It is obvious that $B_{j, i}(\theta) \geq 0 ; i=1,2, \ldots, n-1$, $j=0,1,2,3$. (b) $\sum_{j=0}^{3} B_{j, i}(\theta)=(1-\sin \theta)^{2}(1+\sin \theta)+\sin \theta\left(1-\sin ^{2} \theta\right)+$ $\cos \theta\left(1-\cos ^{2} \theta\right)+(1-\cos \theta)^{2}(1+\cos \theta)=(1-\sin \theta-$ $\left.\sin ^{2} \theta+\sin ^{3} \theta\right)+\left(\sin \theta-\sin ^{3} \theta\right)+\left(\cos \theta-\cos ^{3} \theta\right)+(1-$ $\left.\cos \theta-\cos ^{2} \theta+\cos ^{3} \theta\right)=\left(1-\sin ^{2} \theta\right)+\left(1-\cos ^{2} \theta\right) \equiv 1$.

(c) For $\quad i=1,2, \ldots, n-1, \quad$ as $\quad \cos \theta=\sin \left(\frac{\pi}{2}-\theta\right)$ and $\sin \theta=\cos \left(\frac{\pi}{2}-\theta\right)$.

$$
\begin{aligned}
B_{0, i}(\theta)= & (1-\sin \theta)^{2}(1+\sin \theta)=\left(1-\cos \left(\frac{\pi}{2}-\theta\right)\right)^{2} \\
& \left(1+\cos \left(\frac{\pi}{2}-\theta\right)\right)=B_{3, i}\left(\frac{\pi}{2}-\theta\right) \\
B_{1, i}(\theta)= & \sin \theta\left(1-\sin ^{2} \theta\right)=\cos \left(\frac{\pi}{2}-\theta\right)\left(1-\cos ^{2}\left(\frac{\pi}{2}-\theta\right)\right) \\
= & B_{2, i}\left(\frac{\pi}{2}-\theta\right) . \\
B_{2, i}(\theta)= & \cos \theta\left(1-\cos ^{2} \theta\right)=\sin \left(\frac{\pi}{2}-\theta\right)\left(1-\sin ^{2}\left(\frac{\pi}{2}-\theta\right)\right) \\
= & B_{1, i}\left(\frac{\pi}{2}-\theta\right) . \\
B_{3, i}(\theta)= & (1-\cos \theta)^{2}(1+\cos \theta)=\left(1-\sin \left(\frac{\pi}{2}-\theta\right)\right)^{2} \\
& \left(1+\sin \left(\frac{\pi}{2}-\theta\right)\right)=B_{0, i}\left(\frac{\pi}{2}-\theta\right) .
\end{aligned}
$$

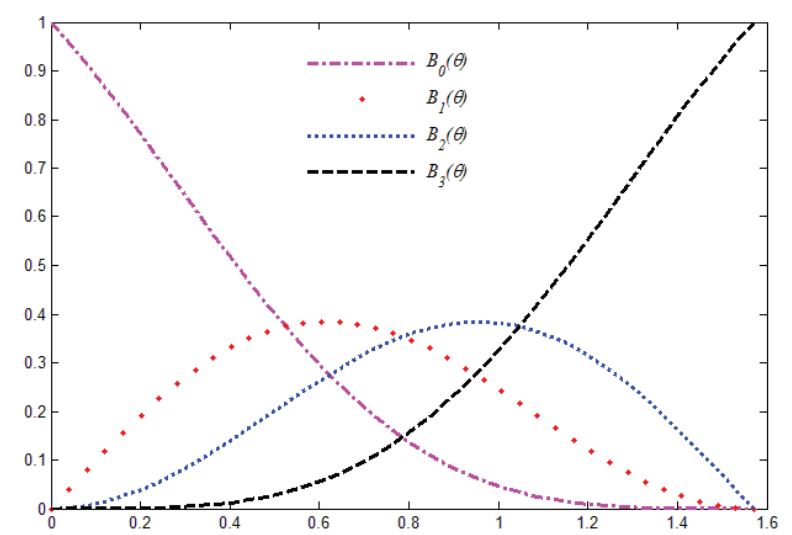

Figure 1: Cubic trigonometric basis functions

\section{Cubic trigonometric spline function}

For a given set of data $\left\{\left(x_{i}, f_{i}\right), i=1, \ldots, n\right\}$ where $x_{1}<x_{2}<\cdots<x_{n}$, a piecewise cubic trigonometric spline function $S(x)$ is defined as follows:

$$
\begin{aligned}
\left.S\right|_{\left[x_{i}, x_{i+1}\right]}(x) & =S_{i}(x)=\sum_{j=0}^{3} B_{j, i}(\theta) P_{j}^{*}, \quad \forall x \in\left[x_{i}, x_{i+1}\right], \\
i & =1, \ldots, n-1
\end{aligned}
$$


where $\quad B_{j, i}(\theta) ; i=1,2, \ldots, n-1, \quad j=0,1,2,3 \quad$ are the trigonometric cubic basis functions defined in equation (1), and $P_{j} ; j=0,1,2,3$ are the control points. Applying the $\mathrm{C}^{1}$ conditions: $S\left(x_{i}\right)=f_{i}$, $S\left(x_{i+1}\right)=f_{i+1}, S^{\prime}\left(x_{i}\right)=d_{i}, S^{\prime}\left(x_{i+1}\right)=d_{i+1}$, the cubic trigonometric function (2) takes the form:

$$
\begin{aligned}
S_{i}(x)= & (1-\sin \theta)^{2}(1+\sin \theta) f_{i}+\sin \theta\left(1-\sin ^{2} \theta\right)\left(f_{i}+\frac{\pi}{2} h_{i} d_{i}\right) \\
& +\cos \theta\left(1-\cos ^{2} \theta\right)\left(f_{i+1}-\frac{\pi}{2} h_{i} d_{i+1}\right)+(1-\cos \theta)^{2} \\
& (1+\cos \theta) f_{i+1}, \quad \forall x \in\left[x_{i}, x_{i+1}\right], i=1, \ldots, n-1 . \quad \ldots(3)
\end{aligned}
$$

Here, the values of $d_{i}$ may be given or approximated by using any approximation scheme such as arithmetic mean, geometric mean and harmonic mean approximations (Sarfraz et al., 1997). In this work the arithmetic mean approximation is utilised for this purpose. As the values of $d_{i}$ in cubic trigonometric spline function (3) is viewed fixed on end points of the interval $\left[x_{i}, x_{i+1}\right]$, the flexible values of these $d_{i}$ s can be obtained by applying $G C^{1}$ conditions, which are defined as follows:

$$
\begin{aligned}
& S_{i-1}^{\prime}\left(x_{i}\right)=K_{1} S_{i}^{\prime}\left(x_{i}\right) \forall i, K_{1}=\frac{\gamma_{i}}{\alpha_{i}+\beta_{i}} \neq 1 \\
& S_{i}^{\prime}\left(x_{i+1}\right)=K_{2} S_{i+1}^{\prime}\left(x_{i+1}\right) \forall i, K_{2}=\frac{\omega_{i}}{\vartheta_{i}+\rho_{i}} \neq 1
\end{aligned}
$$

i.e.

$d_{i} \rightarrow \frac{\gamma_{i}}{\alpha_{i}+\beta_{i}} d_{i}, \quad d_{i+1} \rightarrow \frac{\omega_{i}}{\vartheta_{i}+\rho_{i}} d_{i+1}$,

where $\alpha_{i}, \beta_{i}, \gamma_{i}, \vartheta_{i}, \rho_{i}, \omega_{i} \geq 0 ; i=1, \ldots, n$, are the parameters. The trigonometric cubic spline function (3) is transformed into $G C^{1}$ trigonometric cubic spline function described as:

$\left.S\right|_{\left[x_{i}, x_{i+1}\right]}(x)=S_{i}(x)=\sum_{j=0}^{3} B_{j, i}(\theta) P_{j}, \quad \forall x \in\left[x_{i}, x_{i+1}\right]$

where

$P_{0}=f_{i}, P_{1}=f_{i}+\frac{\pi}{2} \frac{\gamma_{i}}{\alpha_{i}+\beta_{i}} h_{i} d_{i}, P_{2}=f_{i+1}-\frac{\pi}{2} \frac{\omega_{i}}{\vartheta_{i}+\rho_{i}} h_{i} d_{i+1}, P_{3}=f_{i+1}$; $i=1,2, \ldots, n-1$.

Theorem 2. The $G C^{1}$ cubic trigonometric spline function (4) has the following properties:

a. Terminal properties: for each subinterval $I_{i}=\left[x_{i}, x_{i+1}\right] ; i=1, \ldots, n-1$

$$
\begin{aligned}
& S\left(x_{i}\right)=P_{0} \\
& S\left(x_{i+1}\right)=P_{3} \\
& S^{\prime}\left(x_{i}\right)=\frac{\pi}{2} h_{i}\left(P_{0}-P_{1}\right) \\
& S^{\prime}\left(x_{i+1}\right)=\frac{\pi}{2} h_{i}\left(P_{3}-P_{2}\right)
\end{aligned}
$$

where $S^{\prime}$ presents the derivative of the cubic trigonometric spline function $S$ with respect to variable $x$.

b. Convex hull property: the entire segment of piecewise $G C^{1}$ cubic trigonometric spline function must lie inside the control polygon spanned by $P_{0}, P_{1}, P_{2}$ and $P_{3}$.

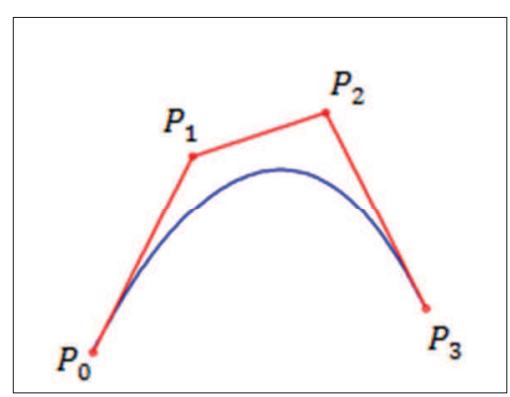

Figure 2: Graphical view of convex hull property for the proposed cubic trigonometric spline function

c. Variation diminishing property: it can be stated as the curve has no more intersections with any plane than does the control polygon.

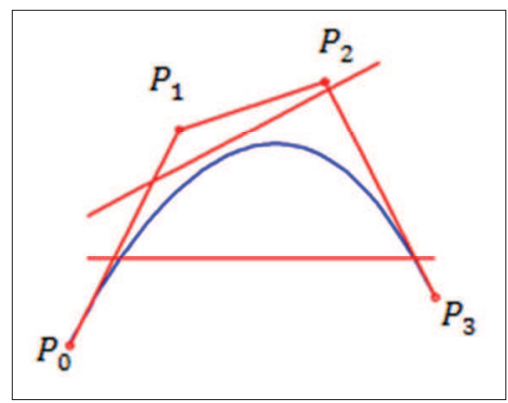

Figure 3: Graphical view of variation diminishing property for the proposed cubic trigonometric spline function

Figures 2 and 3, respectively show the graphical view of convex hull property and the variation diminishing property for the proposed cubic trigonometric spline function.

\section{Implementation strategy}

This section describes the implementation strategy of the proposed cubic trigonometric spline function to still digital images. Since digital images are formed from two dimensional data, their physical size is controlled by the number of sample points and sample spacing. 
The proposed trigonometric spline function was applied to the selected image data in two dimensional spatial domain using tensor products.

Let us consider a set of image sample data, $\left\{\left(x_{i}, y_{j}, f_{i, j}\right) ; i=1, \ldots\right.$, m and $\left.j=1, \ldots, n\right\}$ chosen from an arbitrary rectangular domain in the $x y$-plane where $x$ and $y$ are non-negative real valued coordinates and $f_{i, j}$ presents the image intensity or pixel value at the spatial location $\left(x_{i}, y_{j}\right)$. Over each rectangular sub-division $\left[x_{i}, x_{i+1}\right] \times\left[y_{j}, y_{j+1}\right]$, the proposed cubic trigonometric spline function $S(x, y)$ will be defined as follows:

$$
\begin{gathered}
S_{i, j}(x, y)=\sum_{k=0}^{3} \sum_{l=0}^{3} B_{k, i}(\theta) B_{l, j}(\varphi) P_{i+k-1, j+l-1}, \\
\forall x \in\left[x_{i}, x_{i+1}\right], y \in\left[y_{j}, y_{j+1}\right]
\end{gathered}
$$

where $\theta=\frac{\left(x-x_{i}\right)}{h_{i}}$ and $\varphi=\frac{\left(y-y_{j}\right)}{\hat{h}_{j}}$, whereas $h_{i}=x_{i+1}-x_{i}>0$ and $\hat{h}_{j}=y_{j+1}-y_{j}>0$, for $i=1,2, \ldots, m$ and $j=1,2, \ldots, n$.

\section{RESULTS AND DISCUSSION}

Three different medical test images, named as CT scanspine, MRI-brain 1 and MRI-brain 2, of size $512 \times$ 512 with 8 bits per pixel were selected to compare the performance of the proposed scheme with conventional techniques including bilinear and bicubic schemes for medical image interpolation. The proposed scheme was applied on the selected test images to get the resulting interpolated image. First the images were down-sampled to $256 \times 256$ and then reproduced to their original size of $512 \times 512$. Since quality is a major concern with medical images, the quality of the resulting image in contrast to the original test image was evaluated in terms of peak signal-to-noise ratio (PSNR) (Fevralev et al., 2011), root mean square error (RMSE) (Lalitha \& Latte, 2011) and structural similarity index measure (SSIM) (Wang et al., 2004).

PSNR is the most commonly used objective metric and is very simple to calculate. It is usually expressed in units of decibel $(\mathrm{dB})$. On comparison of the resulting image with the test image, normally a higher PSNR value indicates a high quality resulting image and a low PSNR value indicates a low quality resulting image. In some cases a resulting image may appear to be closer to the test image although it has a low PSNR value. RMSE is a measure of the residuals (differences) of the test and the resulting images. It is used to combine the residuals into a single measure of analytical power.
SSIM, an objective image quality metric, is based on structure similarity information of both the test and resulting images. It is the most powerful quality metric among other conventional quality metrics. SSIM is a decimal fraction between 0 and 1 , where value 1 is only reachable in the case of two identical sets of image data. Mathematical descriptions of each of these metrics are presented as follows:

$$
\begin{aligned}
& P S N R=10 \log \left(\frac{2^{M}-1}{\frac{1}{N} \sum_{i} \sum_{j}[Y(i, j)-X(i, j)]^{2}}\right) \\
& R M S E=\sqrt{\frac{1}{N} \sum_{i} \sum_{j}[Y(i, j)-X(i, j)]^{2}} \\
& \text { SSIM }=\frac{\left(2 \mu_{x} \mu_{y}+C_{1}\right)\left(2 \sigma_{x y}+C_{2}\right)}{\left(\mu_{x}^{2}+\mu_{y}^{2}+C_{1}\right)\left(\sigma_{x}^{2}+\sigma_{y}^{2}+C_{2}\right)}
\end{aligned}
$$

where $N$ is the number of pixels in the image and $M$ is the number of bits, which is used to quantise intensity values of the image. $X$ presents the test image whereas $Y$ is the resulting image. $\mu_{x}$ and $\mu_{y}$ are the mean intensities of $X$ and $Y$, respectively whereas $\sigma_{x}$ and $\sigma_{y}$ are the standard deviations used in the estimation of the contrast. $\sigma_{x y}$ corresponds to the covariance and $C_{1}, C_{2}$ are constants to avoid instability.

Figures 4 to 6 show the graphical representation of PSNR, RMSE and SSIM versus bite per pixel for the test images. From Figure 4, one can see that the bilinear scheme produces the lowest PSNR. Likewise, the proposed scheme has better PSNR values than both bilinear and bicubic schemes. In Figures 5 and 6 the bilinear scheme depicts the highest RMSE and lowest SSIM values, respectively, whereas the results attained by the proposed scheme are better than both bilinear and bicubic schemes. Table 1 gives the results obtained from

Table 1: Quantitative analysis for the test images in terms of PSNR (dB), RMSE and SSIM

\begin{tabular}{lllll}
\hline & & Bilinear & Bicubic & Proposed \\
\hline \multirow{2}{*}{ CT scan-spine } & PSNR (dB) & 25.6687 & 26.2107 & 26.2459 \\
& RMSE & 0.05210 & 0.04890 & 0.04870 \\
& SSIM & 0.89020 & 0.91970 & 0.92290 \\
MRI-brain 1 & PSNR (dB) & 26.5316 & 26.7661 & 26.7711 \\
& RMSE & 0.04710 & 0.04590 & 0.04590 \\
& SSIM & 0.96530 & 0.97420 & 0.97490 \\
MRI-brain 2 & PSNR (dB) & 23.3564 & 24.3569 & 24.8537 \\
& RMSE & 0.06790 & 0.06060 & 0.05720 \\
& SSIM & 0.90100 & 0.92800 & 0.93950 \\
\hline
\end{tabular}


the proposed scheme along with bilinear and bicubic schemes for the test images in term of PSNR (dB), RMSE and SSIM. From the table it can be seen that the proposed scheme produces better results than the other conventional schemes. Figures 7 to 9 show the details for the sequence of the resulting medical images and their
SSIM maps produced using bilinear, bicubic and the proposed image interpolation scheme, respectively. One can easily observe that the edges in the medical images interpolated by the bilinear and bicubic interpolation schemes are comparatively blurred, whereas the results shown in the medical images interpolated by the proposed

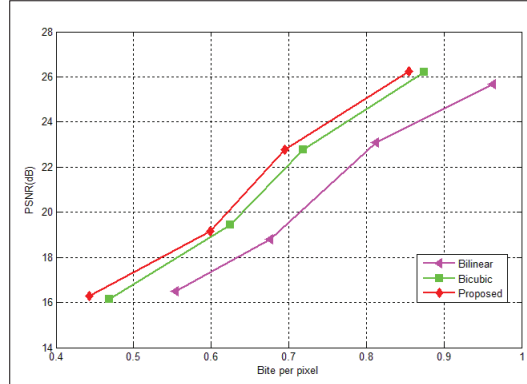

(a)

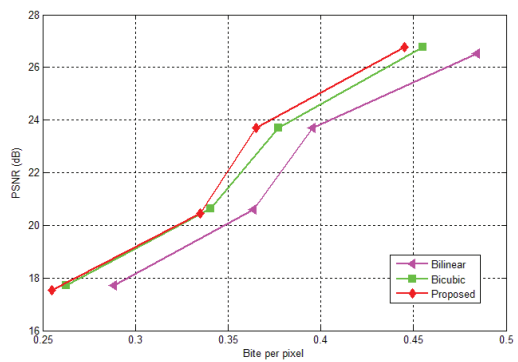

(b)

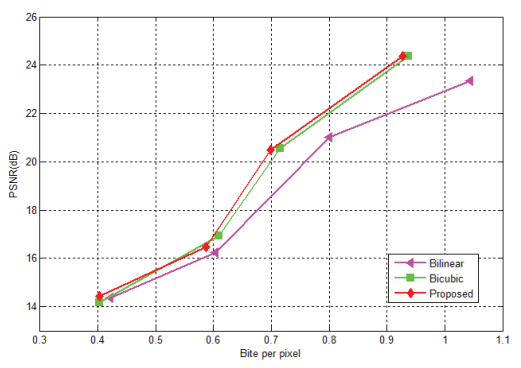

(c)

Figure 4: Graphical representation of objective quality compression, PSNR (dB) vs bite per pixel for the images (a) CT scan-spine;

(b) MRI-brain 1; (c) MRI-brain 2

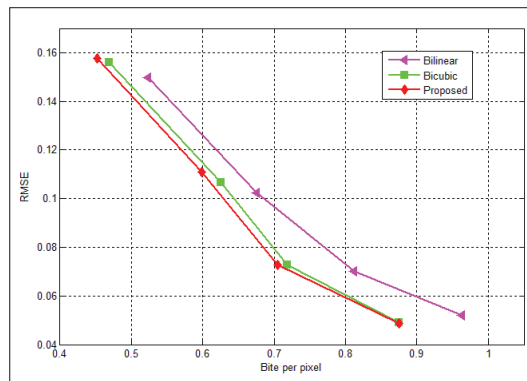

(a)

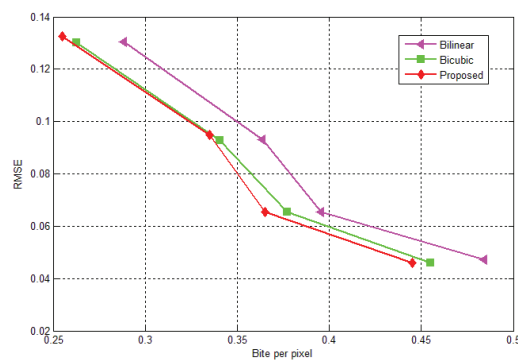

(b)

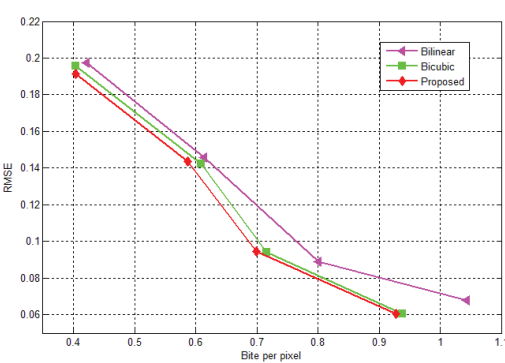

(c)

Figure 5: Graphical representation of objective quality compression, RMSE vs. bite per pixel for the images (a) CT scan-spine; (b) MRI-brain 1; (c) MRI-brain 2

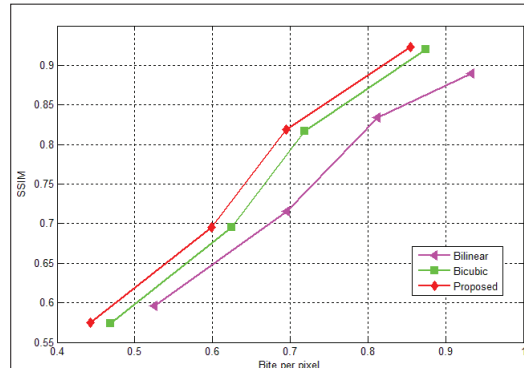

(a)

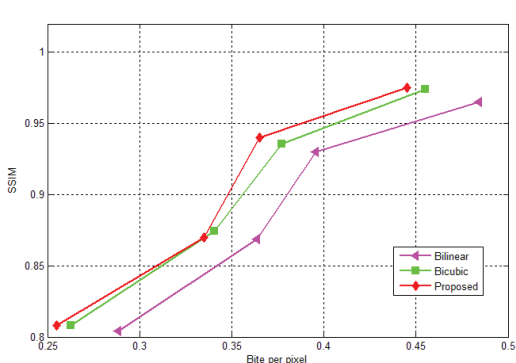

(b)

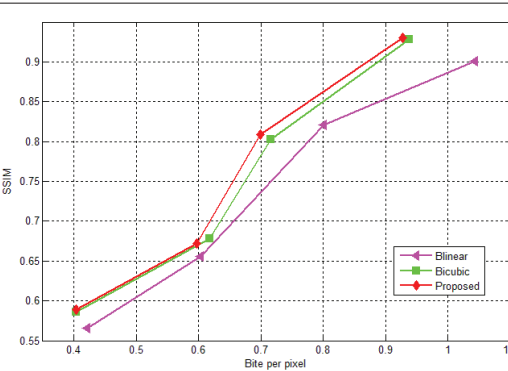

(c)

Figure 6: Graphical representation of objective quality compression, SSIM vs bite per pixel for the images (a) CT scan-spine; (b) MRI-brain 1; (c) MRI-brain 2 


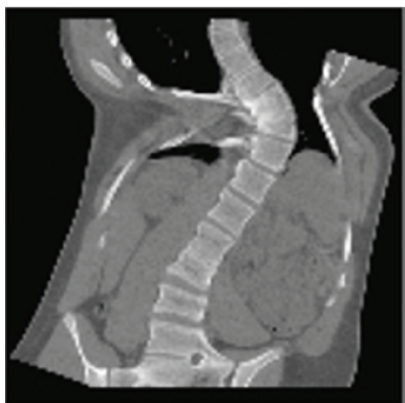

(a)

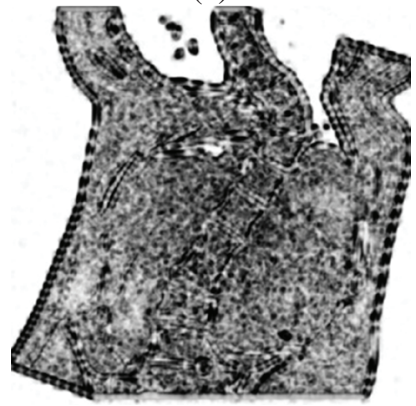

(d)

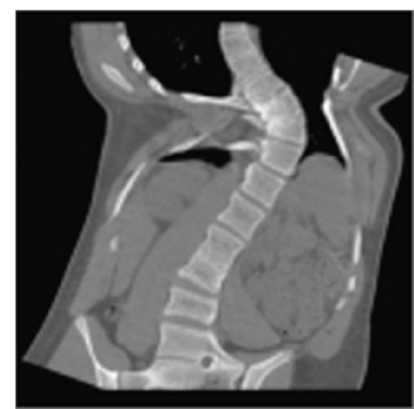

(b)

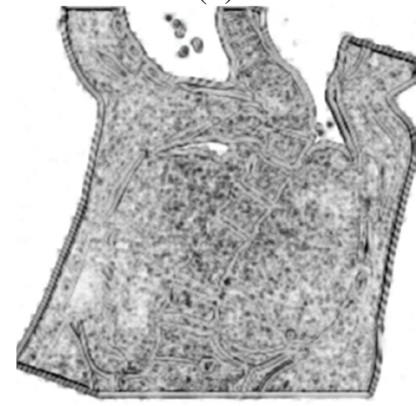

(e)

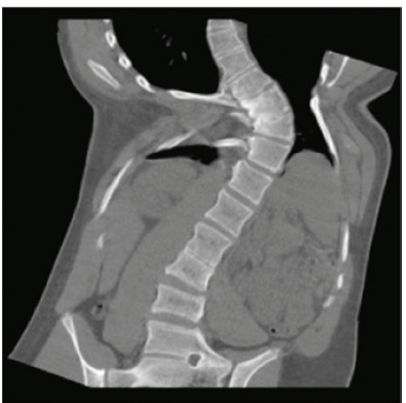

(c)

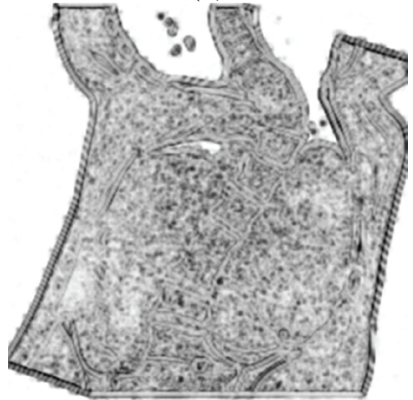

(f)

Figure 7: Subjective quality comparison for a selected CT scan-spine image (a) bilinear; (b) bicubic; (c) proposed, SSIM maps where brightness indicates the magnitude of the SSIM index; (d) bilinear; (e) bicubic; (f) proposed

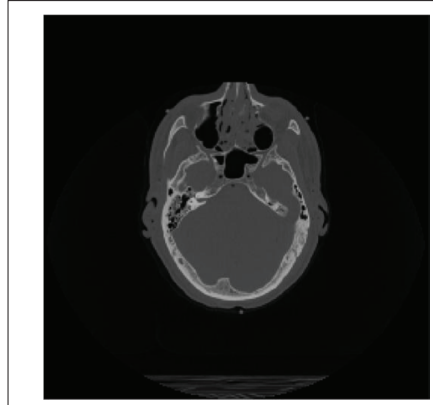

(a)

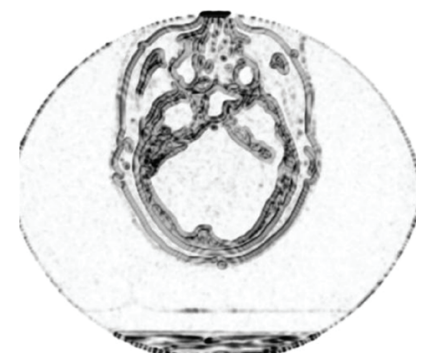

(d)

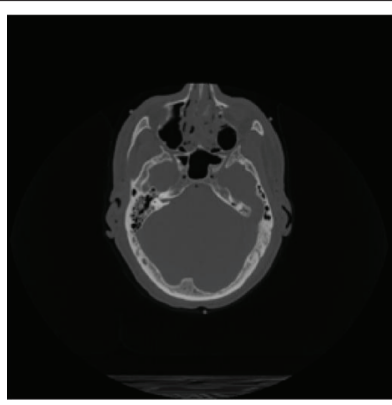

(b)

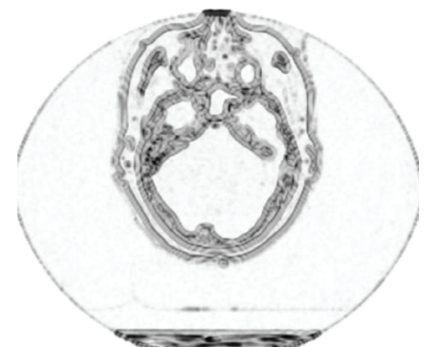

(e)

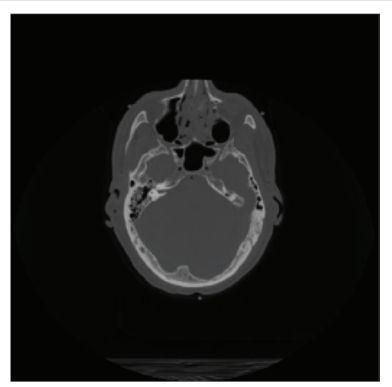

(c)

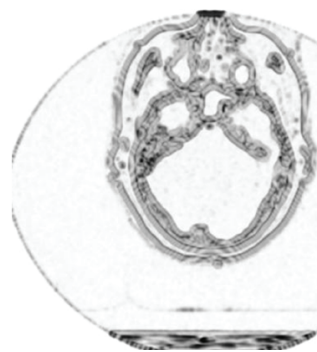

(f)

Figure 8: Subjective quality comparison for a selected MRI-brain 1 image (a) bilinear; (b) bicubic; (c) proposed, SSIM maps where brightness indicates the magnitude of the SSIM index; (d) bilinear; (e) bicubic; (f) proposed 


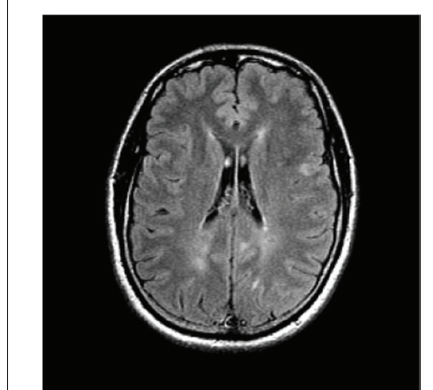

(a)

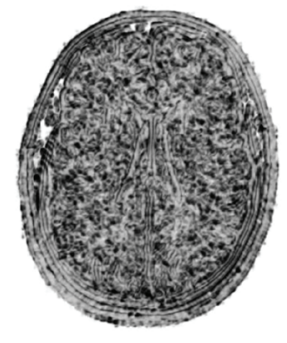

(d)

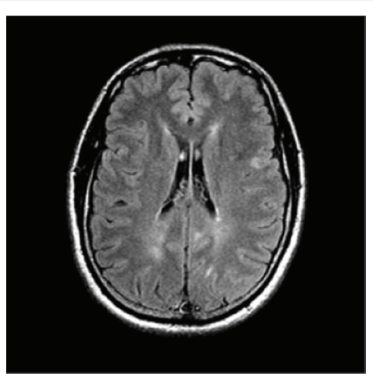

(b)

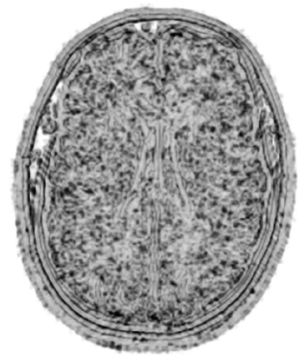

(e)

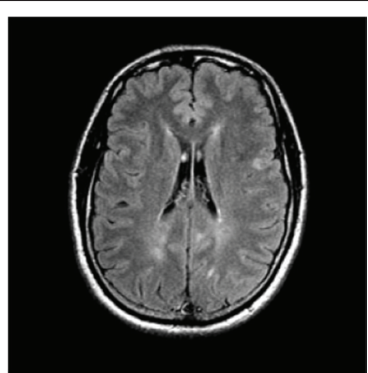

(c)

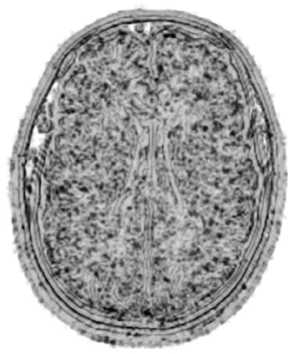

(f)

Figure 9: Subjective quality comparison for a selected MRI-brain 2 image (a) bilinear; (b) bicubic; (c) proposed, SSIM maps where brightness indicates the magnitude of the SSIM index; (d) bilinear; (e) bicubic; (f) proposed

Table 2: PSNR (dB) and SSIM measurements for the test images with different values of quality control parameters

\begin{tabular}{|c|c|c|c|c|c|c|c|c|}
\hline & \multicolumn{2}{|c|}{$\vartheta_{i}=1, \rho_{i}=1, \omega_{i}=1$} & \multicolumn{2}{|c|}{$\vartheta_{i}=1, \rho_{i}=3, \omega_{i}=1$} & \multicolumn{2}{|c|}{$\vartheta_{i}=1, \rho_{i}=3, \omega_{i}=3$} & \multicolumn{2}{|c|}{$\vartheta_{i}=3, \rho_{i}=3, \omega_{i}=1$} \\
\hline & PSNR (dB) & SSIM & PSNR (dB) & SSIM & PSNR (dB) & SSIM & PSNR (dB) & SSIM \\
\hline \multicolumn{9}{|l|}{ CT scan-spine } \\
\hline$\alpha_{\mathrm{i}}=1, \beta_{\mathrm{i}}=1, \gamma_{\mathrm{i}}=1$ & 26.6819 & 0.7616 & 26.7386 & 0.7623 & 26.4330 & 0.7551 & 26.7120 & 0.7611 \\
\hline$\alpha_{\mathrm{i}}=1, \beta_{\mathrm{i}}=3, \gamma_{\mathrm{i}}=1$ & 26.5273 & 0.7610 & 26.6513 & 0.7630 & 26.2184 & 0.7530 & 26.6504 & 0.7623 \\
\hline$\alpha_{\mathrm{i}}=1, \beta_{\mathrm{i}}=3, \gamma_{\mathrm{i}}=3$ & 26.6687 & 0.7567 & 26.6524 & 0.7557 & 26.4853 & 0.7514 & 26.6032 & 0.7541 \\
\hline$\alpha_{\mathrm{i}}=3, \beta_{\mathrm{i}}=3, \gamma_{\mathrm{i}}=1$ & 26.4418 & 0.7594 & 26.5878 & 0.7619 & 26.1191 & 0.7514 & 26.5937 & 0.7613 \\
\hline \multicolumn{9}{|l|}{ MRI-brain 1} \\
\hline$\alpha_{i}=1, \beta_{i}=1, \gamma_{i}=1$ & 26.9080 & 0.8555 & 26.8736 & 0.8526 & 26.8922 & 0.8509 & 26.8922 & 0.8509 \\
\hline$\alpha_{i}=1, \beta_{i}=3, \gamma_{i}=1$ & 26.8721 & 0.8531 & 26.8574 & 0.8523 & 26.8370 & 0.8465 & 26.8370 & 0.8465 \\
\hline$\alpha_{i}=1, \beta_{i}=3, \gamma_{i}=3$ & 26.8965 & 0.8507 & 26.8415 & 0.8455 & 26.9007 & 0.8483 & 26.9007 & 0.8483 \\
\hline$\alpha_{i}=3, \beta_{i}=3, \gamma_{i}=1$ & 26.8506 & 0.8508 & 26.8422 & 0.8506 & 26.8090 & 0.8435 & 26.8090 & 0.8435 \\
\hline \multicolumn{9}{|l|}{ MRI-brain 2} \\
\hline$\alpha_{\mathrm{i}}=1, \beta_{\mathrm{i}}=1, \gamma_{\mathrm{i}}=1$ & 24.0500 & 0.76533 & 24.9526 & 0.76467 & 24.0357 & 0.76540 & 24.8967 & 0.76429 \\
\hline$\alpha_{\mathrm{i}}=1, \beta_{\mathrm{i}}=3, \gamma_{\mathrm{i}}=1$ & 24.9750 & 0.76480 & 24.9196 & 0.76428 & 24.9229 & 0.76474 & 24.8798 & 0.76399 \\
\hline$\alpha_{\mathrm{i}}=1, \beta_{\mathrm{i}}=3, \gamma_{\mathrm{i}}=3$ & 24.0032 & 0.76525 & 24.8668 & 0.76442 & 24.0301 & 0.76548 & 24.8011 & 0.76405 \\
\hline$\alpha_{\mathrm{i}}=3, \beta_{\mathrm{i}}=3, \gamma_{\mathrm{i}}=1$ & 24.9254 & 0.76449 & 24.8843 & 0.76402 & 24.8582 & 0.76437 & 24.8489 & 0.76377 \\
\hline
\end{tabular}

scheme indicates smooth edges with better subjective quality than the others.

To investigate the behaviour of the proposed scheme with different values of quality control parameters in terms of PSNR and SSIM, an arbitrary collection of $248 \times 248$ pixels of the test image is selected. Table 2 shows the values of the corresponding PSNR and SSIM for different values of quality control parameters using the proposed scheme. 


\section{CONCLUSION}

In this paper, a piecewise cubic trigonometric spline scheme was proposed for medical image interpolation and compared with other conventional techniques. The proposed scheme uses multiple parameters to achieve the desired quality of medical images (Table 2). Here three medical test images were selected for experimental work and the results were collected in terms of three different objective quality metrics PSNR, RMSE and SSIM, along with their graphical representation for the selected test images. The experimental results show that the proposed scheme yields comparatively better results than the other conventional techniques both in objective and subjective quality metrics.

\section{REFERENCES}

1. Atoui H., Miguet S. \& Sarrut D. (2006). A fast morphinbased interpolation for medical images: application to conformal radiotherapy. Image Analysis Stereology 25: $95-103$.

DOI: https://doi.org/10.5566/ias.v25.p95-103

2. Fevralev D.V., Ponomarenko N.N., Lukin V.V., Abramov S.K., Egiazarian K.O. \& Astola J.T. (2011). Efficiency analysis of color image filtering. EURASIP Journal on Advance in Signal Processing 1(44): 1 - 19.

3. Hussain M.Z., Hussain M. \& Waseem A. (2014). Shapepreserving trigonometric functions. Computation and Applied Mathematics 33: 411 - 431.

DOI: https://doi.org/10.1007/s40314-013-0071-1

4. Ibraheem F., Hussain M., Hussain M.Z. \& Bhatti A.A. (2012). Positive data visualization using trigonometric function. Journal of Applied Mathematics 2012: 247120.
DOI: https://doi.org/10.1155/2012/247120

5. Lalitha Y.S. \& Latte M.V. (2011). A novel approach noise filtration for MRI image sample in medical image processing. International Journal of Computer Science and Communication 2(2): 359 - 363.

6. Lau P.Y. \& Ozawa S. (2005). A multi parameter hierarchical representation using region-based estimation model for detecting tumor in T2-weighted MRI brain images. Malaysian Journal of Computer Science 18: 1 - 19.

7. Leng J., Xu G. \& Zhang Y. (2013). Medical image interpolation based on multi-resolution registration. Computer and Mathematics with Applications 66: 1 - 18. DOI: https://doi.org/10.1016/j.camwa.2013.04.026

8. Meijiering E.H.W. (2000). Spline interpolation in medical imaging: comparison with other convolutionbased approaches. Proceedings of EUSIPCO 2000, Tenth European Signal Processing Conference, 4 - 8 September, Tampere, Finland, pp. 1989 - 1996.

9. Qi G., Kuanquan W., Yongfeng Y. \& Yongtian Y. (2009). Application of trigonometric spline wavelets in ECG detection. Chinese Journal of Electronics 18(1): 117 - 119.

10. Sarfraz M., Butt S. \& Hussain M.Z. (1997). Surfaces for the visualization of scientific data preserving monotonicity. Proceedings of the IMA Mathematics for Surfaces VII (eds. T.N.T. Goodman \& R. Martin), September 2 - 5, Dundee, UK, pp. $479-495$.

11. Unser M. (2002). Spline: a perfect fit for medical imaging. Proceedings of the SPIE International Symposium on Medical Imaging: Image Processing (MI'02), volume 4684, February 24 - 28, San Diego CA, USA, pp. 225 - 236. DOI: https://doi.org/10.1117/12.467162

12. Wang Z., Bovik A., Sheikh H. \& Simoncelli E. (2004). Image quality assessment: from error visibility to structural similarity. IEEE Transactions on Image Processing 3(4): $600-612$.

DOI: https://doi.org/10.1109/TIP.2003.819861 